\title{
Is Elevated Lipase in Covid-19 Patients Due to Acute Pancreatitis? - A Study from Punjab, India
}

\author{
Shreya Garg' ${ }^{1}$ Sushil Garg², Anju Kansal ${ }^{3}$, Afreen Kansal ${ }^{4}$ \\ ${ }^{1}$ Guru Gobind Singh Medical College and Hospital, Faridkot, Punjab, India, ${ }^{2}$ Department of Anaesthesia and \\ Critical Care, Mediwin Hospital, Bathinda, Punjab, India, ${ }^{3}$ Community Health Centre, Sangat, Bathinda, \\ Punjab, India, ${ }^{4}$ St Joseph College, (Autonomous) Bangalore, Karnataka, India.
}

\section{ABSTRACT}

\section{BACKGROUND}

Since the outbreak of coronavirus disease (Covid-19) many patients have presented with a variety of other symptoms along with primary respiratory symptoms. The increased reporting of these extra-pulmonary symptoms has made it necessary for the clinicians to establish a proper cause and relation between coronavirus disease 2019 (Covid-19) and extra-pulmonary symptoms. This study was undertaken to assess if the cause of elevated lipase levels in Covid-19 patients was due to acute pancreatitis.

\section{METHODS}

A retrospective observational study was performed at a local critical care ICU facility in Bathinda, India (MediWin Hospital, Bathinda, Punjab). RT - PCR, Rapid antigen test, CT scans were done to establish the Covid-19 status. Clinical signs and symptoms, CT scans were used to confirm the diagnosis of acute pancreatitis. MRI was preferred in patients with deranged renal function test.

\section{RESULTS}

Lipase test was done in 62 patients and the levels were found elevated in 52 patients. 40 patients were found to have symptoms of the spectrum of acute pancreatitis. 10 patients had symptoms along with signs of ${ }^{1}$ severe inflammatory response syndrome. Imaging was advised in these 10 patients with ${ }^{1}$ moderate to severe presentation of acute pancreatitis. 9 patients were advised CT scans which had no significant findings. 1 patient with deranged renal function test was advised ${ }^{2}$ MRI scan which showed mildly diffusely bulky pancreas. Mild peripancreatic fat stranding and fluid collection, suggestive of acute interstitial pancreatitis. It was associated with poor outcomes and lead to the patient's death eventually.

\section{CONCLUSIONS}

Hyperlipasemia led to acute pancreatitis in $1(1.6 \%)$ patient. It was associated with poor outcome and caused eventual death of the patient. The prospects of a Covid-19 patient developing Hyperlipasemia due to acute pancreatitis were found to be statistically insignificant.

\section{KEY WORDS}

Coronavirus, Hyperlipasemia, Pancreatitis, Lipase, RT - PCR, Rapid Antigen Test.
Corresponding Author: Dr. (Sqn Ldr) Sushil Garg, Mediwin Hospital, St No. 1, Ajit Road, Bathinda, Punjab, India.

E-mail: drsushilgarg32@gmail.com

DOI: $10.14260 / \mathrm{jemds} / 2021 / 563$

How to Cite This Article:

Garg S, Garg S, Kansal A, et al. Is elevated lipase in Covid-19 patients due to acute pancreatitis? - a study from Punjab, India. J Evolution Med Dent Sci 2021;10(33):27612764, DOI: 10.14260/jemds/2021/563

Submission 02-04-2021,

Peer Review 05-06-2021,

Acceptance 11-06-2021,

Published 16-08-2021.

Copyright (C) 2021 Shreya Garg et al. This is an open access article distributed under Creative Commons Attribution License [Attribution 4.0 International (CC BY 4.0)] 


\section{BACKGROUND}

Coronavirus is an RNA virus that causes mainly respiratory tract infection in birds and mammals. In 2019, coronavirus was reported to cause lethal pneumonia like infection in humans; the first case was reported in Wuhan, China. ${ }^{3}$ On February 11, 2020, the World Health Organization (WHO) officially named this novel coronavirus pneumonia as coronavirus disease 2019 (Covid-19), whereas the International Committee on Taxonomy of Viruses has named it as severe acute respiratory syndrome coronavirus 2 (SARS CoV-2). On 11 March, WHO declared that Covid-19 should be characterized as a pandemic. ${ }^{4}$ India has reported a total of 1,08,26,363 Covid-19 cases till 1 Feb 2021 of which $1.37 \%$ cases are active, and $97.19 \%$ have been discharged and 1.43 $\%$ resulted in fatality. According to ${ }^{5}$ the Lancet, the earliest data of onset of symptoms was on 1 December 2019.6 Symptoms of Covid-19 are variable ranging from mild symptoms like headache, fever, sore throat, nasal congestion to loss of smell, breathing difficulties etc. ${ }^{7} \mathrm{~A}$ total of $81 \%$ of cases in the JAMA study were classified as mild, meaning they did not result in pneumonia or resulted in only mild pneumonia. Fourteen percent of cases were severe (marked by difficulty breathing), and $5 \%$ were critical (respiratory failure, septic shock, and / or multiple organ dysfunction or failure). Apart from lung, Covid-19 is also reported to affect many other organs. $^{8}$ A report published in ${ }^{9}$ JAMA CARDIO stated that cardiac injury is a common condition among hospitalized patients with Covid-19. Another report published in ${ }^{10}$ International Society of Nephrology found extensive acute tubular injury and endothelial injury pattern. ${ }^{11} \mathrm{~A}$ study at Wuhan, China found incidence of venous thromboembolism in patients with severe novel coronavirus pneumonia as $25 \%$ (20 / 81). ${ }^{12}$ Chaolin Huang et al. reported an increase in inflammatory markers in Covid-19 patients. These markers include leukocyte count, procalcitonin level (PCT), C-reactive protein (CRP), interleukin - 6 (IL - 6), interleukin - 10 (IL - 10), and D - dimers. ${ }^{13}$ Usman Barlass reported that an increase in lipase levels was also seen among Covid-19 patients.

Elevated lipase levels have always been associated with acute pancreatitis. ${ }^{14-19}$ In a report from China it was mentioned that some cases of Covid-19 with increased lipase levels were associated with acute pancreatic injury. Our study was done to assess the association of elevated lipase levels with acute pancreatitis in Covid-19 patients, at a local facility in India.

\section{METHODS}

A retrospective observational study was performed at MediWin Hospital, and ICU facility in Bathinda, Punjab, India. The data collected was of admissions from $1^{\text {st }}$ October 2020 to 8 February 2021. A total of 62 patients were taken and written informed consent was taken from all of them.

\section{Inclusion Criteria}

All patients with positive Covid-19 test and elevated lipase levels were included.

\section{Exclusion Criteria}

Patients with diabetes mellitus, chronic alcoholics, previously diagnosed cases of gall stones, cancer, and elevated triglyceride levels, previous episodes of acute pancreatic injury, pregnant females and children were excluded.

\section{Sample Size Estimation}

Minimum sample size was calculated based on Cochran's sample size formula:

$\mathrm{N}=\left(\mathrm{Z}^{\wedge} 2 \mathrm{pq}\right) / \mathrm{e}^{\wedge} 2$

Where,

$\mathrm{N}=$ sample size

$\mathrm{Z}=95 \%$ confidence interval

$\mathrm{p}=$ prevalence according to study

$\mathrm{q}=100-\mathrm{p}$

$\mathrm{e}=$ margin of error $=5 \%$

Sample size of $62(\mathrm{~N}=62)$ was taken based on the inclusion criteria. 7 of them were previously diagnosed cases of type 2 diabetes mellitus and were excluded. 3 of them were chronic alcoholics and were excluded. Thus, study was done in 52 patients $(\mathrm{N}=52)$.

The patients were diagnosed as Covid positive via nasal swab testing by RT - PCR or rapid antigen test. HRCT chest was done for every patient admitted to the hospital. On the basis of HRCT chest score, patients were categorized into mild, moderate, severe (Table1).20 Each of the 5 lung lobes was visually scored from 0 to 5 as: 0 - no involvement; $1-<5 \%$ involvement; $2-25 \%$ involvement; $3-26 \%-49 \%$ involvement; $4-50 \%-75 \%$ involvement; 5 - >75\% involvement. The total CT score was the sum of the individual lobar scores and ranged from 0 (no involvement) to 25 (maximum involvement).

\begin{tabular}{|cc|cc|}
\hline Category & CT Score & No. of Patients & Percentage \\
Mild & $0-8$ & 10 & $16.1 \%$ \\
Moderate & $9-17$ & 30 & $48.3 \%$ \\
Severe & $18-25$ & 22 & $35.4 \%$ \\
\hline \multicolumn{3}{|c|}{ Table 1. Distribution of Participants According to } \\
& HRCT Score and Severity of Covid-19 \\
\hline
\end{tabular}

All patients were investigated for routine investigations (Liver Function Test, Renal Function Test, Complete Blood Count, Blood Sugar, Viral markers), LDH, D - Dimers, CRP along with serum lipase. Level of serum lipase more than the upper limit of normal level (5 - 65U / L) was considered Hyperlipasemia. ${ }^{1}$ Patients were assessed for clinical acute pancreatitis based on signs and symptoms (Table 2) and final diagnosis was made by CECT Abdomen and MRI was preferred in patients with deranged renal function test.

\begin{tabular}{|cc|}
\hline $\begin{array}{c}\text { Symptoms } \\
\text { Upper abdominal pain (with radiation } \\
\text { to back) }\end{array}$ & Signs \\
Vomiting & Elevated heart rate $(>90 / \mathrm{min})$ \\
Nausea & Temperature $>98.6^{\circ} \mathrm{f}$ \\
Bloating & $\left.\mathrm{mm}^{3}\right)$ \\
Fat in stools & \\
\hline Table 2. Symptoms and Signs of Acute Pancreatitis \\
\hline
\end{tabular}


Patients with hyperlipasemia were also divided into mild, moderate, and severe cases according to the Atlanta classification of severity of acute pancreatitis 2012 (Table 3). The symptoms of patients with moderate to severe characteristics were further evaluated.

\begin{tabular}{|c|c|}
\hline \multicolumn{2}{|c|}{ Grades of Severity } \\
\hline Mild acute pancreatitis & $\begin{array}{c}\text { No organ failure } \\
\text { No local or systemic complications }\end{array}$ \\
\hline Moderate acute pancreatitis & $\begin{array}{l}\text { Organ failure that resolves within } 48 \mathrm{~h} \\
\text { (transient organ failure) and / or } \\
\text { Local or systemic complications without } \\
\text { persistent organ failure }\end{array}$ \\
\hline Severe acute pancreatitis & $\begin{array}{c}\text { Persistent organ failure }(>48 \mathrm{~h}) \\
\text { single organ failure } \\
\text { multiple organ failure }\end{array}$ \\
\hline Table 3. Grades of & ty of Acute Pancreatitis \\
\hline
\end{tabular}

\section{Statistical Analysis}

$\chi^{2}$ test was performed using $\mathrm{R}$ to test the significance of the association between hyperlipasemia and acute pancreatitis. The statistical significance was set at 0.05 . ( $\mathrm{P}$ - value $<0.05$ was considered statistically significant).

\section{RESULTS}

There was a total of 62 admissions of Covid-19 positive patients meeting the inclusion criteria and serum lipase test was done in all of them. Of 62 patients, $52(83 \%)$ patients were found to have elevated levels of lipase. Of 52 patients, 40 (76 $\%$ ) patients reported upper abdominal pain radiating to the back, fever $\left(99^{\circ}-102^{\circ} \mathrm{F}\right)$, nausea and vomiting and $10(19.2 \%)$ patients were found to have elevated WBC count (>12000 / $\left.\mathrm{mm}^{3}\right)$, respiratory rate $(>20 / \mathrm{min})$, raised serum creatinine (normal range: $0.84-1.21 \mathrm{mg} / \mathrm{dl}$ ) and increased blood urea nitrogen (BUN) (normal range: $7-20 \mathrm{mg} / \mathrm{dl}$ ). As majority of the patients who were admitted did not show significant signs and symptoms to warrant imaging, hence CT was done in only 10 patients who showed signs and symptoms of moderate to severe grade acute pancreatitis. ${ }^{1} 1$ of the 10 patients had elevated serum creatinine and BUN and could not be administered contrast; therefore, an MRI scan was advised instead.

The CT scan of 9 other patients showed no significant findings. The ${ }^{2}$ MRI scan of 1 patient showed mildly diffusely bulky pancreas. Mild peripancreatic fat stranding and fluid collection, suggestive of acute interstitial pancreatitis. Serum lipase was repeated daily and stayed elevated consistently. The patient's 15-day hospital stay required ventilatory support and was intubated. It eventually resulted in death of the patient. Of 52, 47 patients required artificial respiratory support. 30 patients were put on high flow nasal cannula, 6 required BiPAP / CPAP and 11 had to be intubated. Thus 47 patients required prolonged hospital stay (mean $=12$-day SD \pm 4.88 ). Of 47 patients, 7 had poor outcomes and resulted in death. The poor outcomes in patients with hyperlipasemia and in patients without hyperlipasemia did not show significant difference. We obtained a $\mathrm{P}$ value of 1 (including Yates's Continuity Correction) which is less than the significance level of 0.05 So, we accepted the null hypothesis and concluded that there is no association between hyperlipasemia and acute pancreatitis.

\section{DISCUSSION}

Studies conducted by ${ }^{19}$ Wang et al. in Wuhan, China, suggested an association of elevated lipase levels with acute pancreatitis. They noted that $17 \%$ patients with hyperlipasemia presented with acute pancreatic injury and these patients with pancreatic injury were associated with poor outcomes i.e., they had low oxygen saturation ( $\leq 93 \%$ ) and increased breathing rate of $\geq 30 / \mathrm{min}$. These patients were classified as "seriously ill". However, some recent studies have shown that elevated lipase levels may not be associated with pancreatic injury. The study by ${ }^{21}$ McNabb - Baltar et al. showed hyperlipasemia in $12.9 \%$ patients of which $2.8 \%$ had lipase levels more than 3 times the normal. None of the patients met the diagnostic criteria for acute pancreatitis and hyperlipasemia did not lead to poor outcomes. The authors speculated that the mechanism of lipase elevation was related to other gastroenterology manifestations of the virus including gastritis, enteritis or colitis, rather than a marker of pancreatic injury.

Our study showed 40 patients with symptoms in spectrum of acute pancreatitis ${ }^{1}$ along with hyperlipasemia. 10 patients were labelled as possibly having moderate or severe acute pancreatitis. 9 of them were advised CT scans and 1 was advised MRI scan due to deranged renal function test. The 9 CT scans did not show any significant findings, however, ${ }^{2}$ the MRI findings of the patient fulfilled the diagnostic criteria of acute pancreatitis. The cause of this patient's outcome would have been due to the "The pathological changes in these organs and tissues that may be caused directly by the cytopathic effect (CPE) mediated by SARS-CoV replication in these organs; or indirectly by the systemic toxic reaction due to failure of the respiratory system, or by the harmful immune response and cytokine reaction induced by virus infection" as per the study by Yanqing et al. ${ }^{22}$

Hyperlipasemia can be due to increased hospital stay as the patients who presented were with moderate or severe disease and had to stay in the hospital for longer periods due to severity of the disease or due to its associated complications. ${ }^{23}$ The cause of GI symptoms unrelated to acute pancreatitis could have been due to microbial disruption in the intestine as noted by Sonia Villapo ${ }^{24}$ in her study. SARS-CoV-2 can cause gastrointestinal symptoms, such as vomiting, diarrhoea, or abdominal pain during the early phases of the disease. Intestinal dysfunction induces changes in intestinal microbes and an increase in inflammatory cytokines.

Some studies suggest the use of treatment medications as the cause of nausea, vomiting, diarrhoea, and other GI symptoms seen in Covid-19 patients. The standard treatment protocol in all the patients in our study comprised mainly of remdesivir, methylprednisolone, antibiotics, antifibrotic (pirfenidone) along with symptomatic treatment and nutritional supplements. ${ }^{24}$

Lisa $\mathrm{H}$ Lancaster et al. noted that anti-fibrotic like pirfenidone can cause adverse GI effects like nausea (17.4 per $100 \mathrm{PEY}$ ) and diarrhoea (14.4 per $100 \mathrm{PEY}$ ). [per $100 \mathrm{PEY}$ is equivalent to the frequency at which a physician might expect these treatment emergent adverse effects to occur if 100 patients with IPF were followed for 1 year]. Antiviral remdesivir is also known to cause GI side effects which may be similar to the mild symptoms of acute pancreatitis. ${ }^{25}$ 


\section{CONCLUSIONS}

Our study showed that hyperlipasemia due to acute pancreatitis in Covid-19 positive patients was statistically insignificant but can be clinically significant. Therefore, more studies need to be done in order to get more clarity on the causes of hyperlipasemia in Covid-19 patients. There are several limitations in our report. First, this is a retrospective, single-centre, small sample study. The results may be biased and need to be confirmed by a large sample study. Second, some patients are still being treated in hospital, and the clinical outcome may change.

Data sharing statement provided by the authors is available with the full text of this article at jemds.com.

Financial or other competing interests: None.

Disclosure forms provided by the authors are available with the full text of this article at jemds.com.

Author Contributions - Study design: Shreya Garg, Anju Kansal, Sushil Garg. Clinical data collection: Sushil Garg. Data analysis: Sushil Garg, Shreya Garg, Anju Kansal, Afreen Kansal. Manuscript writing: Shreya Garg, Anju Kansal, Sushil Garg. Statistics: Afreen Kansal. All authors have read and approved the final manuscript.

\section{REFERENCES}

[1] Banks PA, Bollen TL, Dervenis C, et al. Classification of acute pancreatitis--2012: revision of the Atlanta classification and definitions by international consensus. Gut 2013;62(1):102-11.

[2] Miller FH, Keppke AL, Dalal K, et al. MRI of pancreatitis and its complications: part 1, acute pancreatitis. Am J Roentgenol 2004;183(6):1637-44.

[3] Li Y, Wang H, Wang F, et al. Comparison of hospitalized patients with pneumonia caused by COVID-19 and influenza A in children under 5 years. Int J Infect Dis 2020;98:80-3

[4] MoHFW. Home. [cited 2021 Feb 15]. https://www.mohfw.gov.in/

[5] Sun K, Chen J, Viboud C. Early epidemiological analysis of the coronavirus disease 2019 outbreak based on crowdsourced data: a population-level observational study. Lancet Digitit Health 2020;2(4):e201-8.

[6] Coronavirus disease 2019. [cited 2021 Feb 15]. https://en.wikipedia.org/wiki/Coronavirus_disease_201 9\#Signs_and_symptoms

[7] Study of 72,000 COVID-19 patients finds $2.3 \%$ death rate $\begin{array}{llll}\text { CIDRAP } & \text { [cited } & 2021 & \text { Feb }\end{array}$ https://www.cidrap.umn.edu/newsperspective/2020/02/study-72000-covid-19-patientsfinds-23-death-rate

[8] Fan Q, Zhang B, Ma J, et al. Safety profile of the antiviral drug remdesivir: an update. Biomed Pharmacother 2020;130:110532.
[9] Shi S, Qin M, Shen B, et al. Association of cardiac injury with mortality in hospitalized patients with COVID-19 in Wuhan, China. JAMA Cardiol 2020;5(7):802-10.

[10] Su H, Yang M, Wan C, et al. Renal histopathological analysis of 26 postmortem findings of patients with COVID-19 in China. Kidney Int 2020;98(1):219-27.

[11] Cui S, Chen S, Li X, et al. Prevalence of venous thromboembolism in patients with severe novel coronavirus pneumonia. J Thromb Haemost 2020;18(6):1421-4.

[12] Huang C, Wang Y, Li X, et al. Clinical features of patients infected with 2019 novel coronavirus in Wuhan, China. Lancet 2020;395(10223):497-506.

[13] Barlass U, Wiliams B, Dhana K, et al. Marked elevation of lipase in COVID-19 disease: a cohort study. Clin Transl Gastroenterol 2020;11(7):e00215.

[14] Basnayake C, Ratnam D. Blood tests for acute pancreatitis. Aust Prescr 2015;38(4):128-30.

[15] Frossard JL, Steer ML, Pastor CM. Acute pancreatitis. Lancet 2008;371(9607):143-52.

[16] Lippi G, Valentino M, Cervellin G. Laboratory diagnosis of acute pancreatitis: in search of the Holy Grail. Crit Rev Clin Lab Sci 2012;49(1):18-31.

[17] Al-Bahrani AZ, Ammori BJ. Clinical laboratory assessment of acute pancreatitis. Clin Chim Acta 2005;362(1-2):2648.

[18] Hameed AM, Lam VWT, Pleass HC. Significant elevations of serum lipase not caused by pancreatitis: a systematic review. HPB (Oxford) 2015;17(2):99-112.

[19] Wang F, Wang H, Fan J, et al. Pancreatic injury patterns in patients with coronavirus disease 19 pneumonia. Gastroenterology 2020;159(1):367-70.

[20] Pan F, Ye T, Sun P, et al. Time course of lung changes at chest CT during recovery from Coronavirus disease 2019 (COVID-19). Radiology 2020;295(3):715-21.

[21] McNabb-Baltar J, Jin DX, Grover AS, et al. Lipase elevation in patients with COVID-19. Am J Gastroenterol 2020;115(8):1286-8.

[22] Ding Y, He L, Zhang Q, et al. Organ distribution of severe acute respiratory syndrome (SARS) associated coronavirus (SARS-CoV) in SARS patients: implications for pathogenesis virus transmission pathways. J Pathol 2004;203(2):622-30.

[23] Jin DX, Yang AL, Suleiman SL, et al. Tu1424-marked serum lipase elevations are associated with longer hospitalizations in patients with non-pancreatic hyperlipasemia. Gastroenterology 2019;156(6):S1033-4.

[24] Villapol S. Gastrointestinal symptoms associated with COVID-19: impact on the gut microbiome. Transl Res 2020;226:57-69.

[25] Lancaster LH, De Andrade JA, Zibrak JD, et al. Pirfenidone safety and adverse event management in idiopathic pulmonary fibrosis. Eur Respir Rev 2017;26(146):170057. 